\title{
AVBVICVII ferroelectrics as novel materials for phononic crystals
}

\section{Selami Palaz, Oral Oltulu, Amirullah M. Mamedov \& Ekmel Ozbay}

To cite this article: Selami Palaz, Oral Oltulu, Amirullah M. Mamedov \& Ekmel Ozbay (2017) AVBVICVII ferroelectrics as novel materials for phononic crystals, Ferroelectrics, 511:1, 12-21, DOI: 10.1080/00150193.2017.1332966

To link to this article: https://doi.org/10.1080/00150193.2017.1332966

$$
\text { 册 Published online: } 01 \text { Aug } 2017 .
$$

Submit your article to this journal $\pi$

III Article views: 44

Q View related articles $\circlearrowright$

View Crossmark data $\nearrow$ 


\title{
AVBVICVII ferroelectrics as novel materials for phononic crystals
}

\author{
Selami Palaz ${ }^{\mathrm{a}}$, Oral Oltulu ${ }^{\mathrm{a}}$, Amirullah M. Mamedov ${ }^{\mathrm{b}, \mathrm{c}}$, and Ekmel Ozbay ${ }^{\mathrm{b}}$ \\ ${ }^{a}$ Department of Physics, Harran University, Sanliurfa, Turkey; ${ }^{b}$ Nanotechnology Research Center (NANOTAM), \\ Bilkent University, Ankara, Turkey; International Scientific Center, Baku State University, Baku, Azerbaijan
}

\begin{abstract}
In the present work the acoustic band structure of a two-dimensional (2D) phononic crystal (PC) containing a semiconducting ferroelectric AVBVICVII $(A=S b, B i ; B=S$, Se, Te; $C=I, B r$, and $\mathrm{Cl}$ ) was investigated theoretically and numerically by the plane-wave-expansion (PWE) method. Two-dimensional PC with square lattices composed of semiconducting ferroelectric cylindrical rods embedded in the organic/inorganic matrix is studied to find the existence of stop bands for the waves of certain energy. This phononic bandgap - forbidden frequency range - allows sound to be controlled in many useful ways in structures that can act as sonic filters, waveguides or resonant cavities. Phononic band diagram $\omega=\omega(\mathrm{k})$ for a $2 \mathrm{D}$ PC was plotted versus the wavevector $\mathrm{k}$ along the $\Gamma-\mathrm{X}-\mathrm{M}-\Gamma$ path in the square Brillouin zone (BZ). The band diagram shows four stop bands in the wide frequency range. The unusual properties of matrix and ferroelectric properties of AVBVICVII give us ability to control the wave propagation through the $P C$ in over a wide frequency range. We study the $2 D$ composites by solving the basic acoustic wave equation and use Bloch wave analysis to identify the band gaps.
\end{abstract}

\section{ARTICLE HISTORY}

Received 19 June 2016

Accepted 27 March 2017

\section{KEYWORDS}

Phononic crystals; band gaps; metamaterials; ferroelectrics

\section{Introduction}

Phononic crystals (PnCs) and acoustic metamaterials have generated rising scientific interests for very diverse technological applications ranging from sound abatement to ultrasonic imaging to telecommunications to thermal management and thermoelectricity [1]. In recent years great interest arises in the artificial periodic structures that can arise only in the human imagination. Depending on the specification of a specific application that are periodic in 1D, $2 \mathrm{D}$ and $3 \mathrm{D}$, rapid developments in technology have made possible the production of PnCs operating at desired frequencies. Because PnCs can control the behavior of sound, the PnCs are considered to be main elements for future technology, especially in wireless communication, transducer and sensor system [2-6].

PnCs and acoustic metamaterials are artificially structured composite materials that enable manipulation of the dispersive properties of vibrational waves. PnCs perform several important functions, containing anisotropic propagation and acoustic band gaps [7]. These types of phenomenon are quite frequent for surface acoustic wave (SAW), bulk acoustic 
wave (BAW), and plate waves, also called Lamb waves in different PnC structures, respectively. Applications of PnCs, such as damping acoustic vibrations, a phononic lens, a frequency selective filter in the acoustic regime, reflective gratings for SAW waves, waveguides, and resonant cavities were proposed, and they showed great potential and feasibility of applying PnCs in acoustic electronic devices [8-13]. Among these components, one-way phononic waveguides and SAW devices could potentially lead to the design of a novel class of surface wave devices that are widely used in electronics, telecommunication, and acoustic imaging [14].

PnCs are made of periodic distributions of inclusions (polymer in water, narrow band semiconductor in air, etc.) embedded in a matrix. Also, it is well known that with such a structure, band gaps of PnCs may appear and are independent of the direction of propagation of the incident elastic wave. PnCs are designed to control the dispersion of waves through Bragg scattering, the scattering of waves by a periodic arrangement of scatterers with dimensions and periods comparable to the wavelength. Acoustic metamaterials have the added feature of local resonance, and although often designed as periodic structures, their properties do not rely on periodicity. Finally, the study of PnCs and acoustic metamaterials has also extensively relied on a combination of theory and experiments that have shown extraordinary complementarity [15].

Due to their interesting property, the phononic structures have extensive practical applications by supressing sound waves for a given frequency range. Therefore, the application of phononic crystals can be mainly determined by manipulating the regulation performance level of the band gap. The condition of controlling and tuning band gap in conventional research is primarily met by changing the geometry of lattice, filling fraction and orientation of scatterers in a phononic crystal $[16,17]$. Characteristics of phononic crystals can be altered by changing the geometry of the inclusions or by varying the elastic characteristics of the constitutive materials. The form and geometry of the inclusions embedded in a matrix are, in principle, predefined regular shapes such as circle, square, ellipse, hexagon and so on. However, material selection has a great effect to manipulate sound and hypersound frequencies. Therefore, different materials are needed to fabricate phononic structures to obtain reachable frequencies for the energy band gaps from about kilohertz to megahertz range and even to ultra high frequencies (very high-frequency sound, with $\mathrm{f}<1 \mathrm{GHz}$ ) [18].

There has been a growing interest in ferroelectrics of the AVBVICVII type due to the fact that they have some very interesting properties. In antimony sulfur iodide, SbSI, crystals, the temperature of the ferroelectric phase transition is close to $295 \mathrm{~K}$ and they have been attractive for its fundamental research interest and prospective applications in the fields of ferroelectricity, microelectronics and optoelectronics, as microcapacitors, optical valves [19]. Besides, in virtue of these characteristics, SbSI has the highest piezoelectric constant and is more sensitive to external pressure by an order of magnitude [20].

More importantly, all these combined properties favours SbSI for the efficient creation of low pressure piezoelectric elements with high resonant frequency, and high sensitivity. Its potential for use in acoustic sensors has been exploited for a long time.

SbSI proved to be far superior and won out over many familiar ferroelectrics in efficiency. Such properties, therefore, make the crystals amenable for conducting experimental and theoretical investigations. Ferroelectric materials might have a possible future in phononic crtystals as constitutive materials for creating tunable PnCs and smart temperature-tuned devices such as the Lamb wave filters or sensors. [21]. 


\section{Theory}

The wave propagation in a homogeneous medium can be strongly changed by inserting periodically distributed inclusions (single acoustic functional scatter) in a matrix with different mass densities and/or elastic constants, which can give rise to new acoustic dispersions and band structures due to the periodic Bragg scattering from the inclusions. The periodic scattering in this so-called PnCs induces a wave scattering and destructive interferences can appear in some frequency ranges, leading to forbidden band-gaps. Total reflection is then expected in these frequency ranges. PnCs can be generally classified into two main categories: acoustic phononic crystals (APCs) [22,23-25] with a fluid matrix, and elastic phononic crystals (EPCs) with an elastic solid matrix [26]. In this study, we will mainly focus on acoustic phononic crystals since most of the scientific studies has been conducted in this area.

We consider a 2D system consisting of infinitely long cylinders aligned along the $\mathrm{z}$ direction and any material based parameters are independent of $z$ direction. The phononic crystal also extends infinitely in the xy plane. Therefore, the mechanical properties of the phononic crystal vary periodically in the xy plane. Acoustic waves propagate within the solid-fluid phononic crystals as Bloch waves.

It is possible to simplify the problem under certain assumptions by assuming that solid inclusions develop no deformation, such that spontaneous contributions from piezoelectricity can be neglected. After doing that the problem to be solved is very similar to the acoustic wave equation

$$
\rho \frac{\partial^{2} \vec{u}}{\partial t^{2}}=\vec{\nabla} \cdot\left(\rho c_{l}^{2} \vec{\nabla} \cdot \vec{u}\right)
$$

where $c_{l}=\sqrt{C_{11} / \rho}$ is the longitidunal speed of sound, $C_{11}$ is the longitidunal elastic constant, $\rho$ is the mass density of element and $\vec{\nabla}$ is the two dimensional nabla operator. Introducing a scalar quantity such that $\rho \vec{u}=\nabla p(\vec{r}, t)$, where $\mathrm{p}$ is the pressure, equation (1) can be rewritten as

$$
\left(C_{11}\right)^{-1} \frac{\partial^{2} p}{\partial t^{2}}=\vec{\nabla} \cdot\left(\rho^{-1} \nabla p\right)
$$

the pressure $\mathrm{p}$ in equation (2) can be written as

$$
p(\vec{r}, t)=e^{i(\vec{K} \vec{r}-\omega t)} p_{\vec{K}}(\vec{r})
$$

where the second term is a periodic function with the same periodic structure as $\sum_{\vec{G}} p(\vec{G}) e^{i \vec{G} \vec{r}}$, and $\mathrm{K}$ is the Bloch vector. Taking advantage of the two dimensional periodicity in the (xy) plane, the quantities can be expanded in the Fourire series as given below

$$
C_{11}^{-1}(\vec{r})=\sum_{\vec{G}} \tau(\vec{G}) e^{i \overrightarrow{\mathrm{G}} \vec{r}} \text { and } \rho^{-1}(\vec{r})=\sum_{\vec{G}} \sigma(\vec{G}) e^{i \overrightarrow{\mathrm{G}} \vec{r}}
$$


where $\vec{G}=\left(\frac{2 \pi n_{x}}{a}\right) \hat{x}+\left(\frac{2 \pi n_{y}}{a}\right) \hat{y}$ is the $2 \mathrm{D}$ reciprocal lattice vector and $\mathrm{r}$ is the direct lattice vector. The Fourier transforms of these functions

$$
\begin{gathered}
\sigma(\vec{G})= \begin{cases}\rho_{a}^{-1} f+\rho_{b}^{-1}(1-f)=\overline{\rho^{-1}} & \text { for } \vec{G}=0 \\
\left(\rho_{a}^{-1}-\rho_{b}{ }^{-1}\right) F(\vec{G})=\Delta\left(\rho^{-1}\right) F(\vec{G}) & \text { for } \vec{G} \neq 0\end{cases} \\
\tau(\vec{G})= \begin{cases}C_{a}^{-1} f+C_{b}^{-1}(1-f)=\overline{C^{-1}} & \text { for } \vec{G}=0 \\
\left(C_{a}^{-1}-C_{b}^{-1}\right) F(\vec{G})=\Delta\left(C^{-1}\right) F(\vec{G}) & \text { for } \vec{G} \neq 0\end{cases} \\
F(\vec{G})=\frac{1}{A_{c}} \int_{a} e^{-i \vec{G} \vec{r}} d^{2} r=2 f J_{1}(G r) /(G r)
\end{gathered}
$$

where $f$ is the filling fraction of the cylindrical rods with lattice constant a, $F(G)$ is the structure factor, $\mathrm{J}(\mathrm{x})$ is the Bessel function of the first kind of order one. $\mathrm{A}_{\mathrm{c}}$ is the area of a unit cell, and $r_{0}$ is the cylinder's radius. The integral in $F(\mathbf{G})$ is over the cross-sectional area of cylinder of radius $r$. By applying some algebra, one can obtain

$$
\sum_{\vec{G}^{\prime} \neq \vec{G}}\left[\sigma\left(\vec{G}-\vec{G}^{\prime}\right)(\vec{K}+\vec{G}) \cdot(\vec{K}+\vec{G})-\tau\left(\vec{G}-\vec{G}^{\prime}\right) \omega_{K}^{2}\right] p_{\vec{K}}\left(\vec{G}^{\prime}\right)=0 .
$$

Equation 8 is a $\mathrm{NxN}$ matrix equation and it is obtained using a finite number of Fourier components in the expansion. The solution of the secular equation gives the $\omega(\vec{K})$ eigenvalues and $p(\vec{G})$ eigenvectors. For software implementation and numerical calculation, the code written by Elford [27] was used with some modification such that it was executed for $\Gamma-\mathrm{X}$, $\mathrm{X}-\mathrm{M}$ and $\Gamma-\mathrm{M}$ directions to obtain eigenvalues and eigenvectors of the system.

\section{Results and discussion}

Figure 1 shows the 2D phononic crystal array structure. The cylindrical materials and their environment around them can be set to different materials. Numerical calculation in this

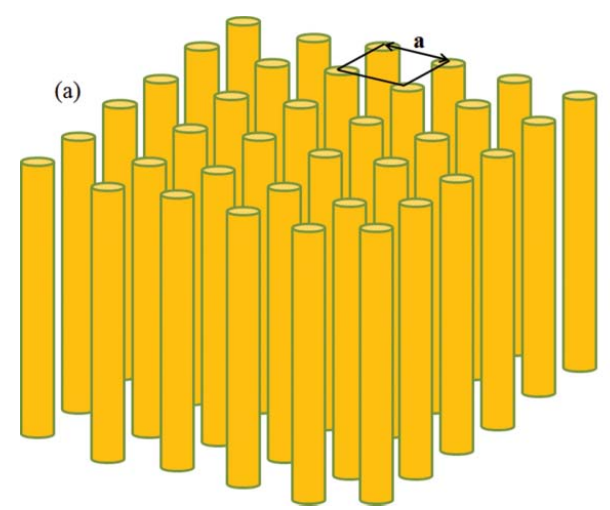

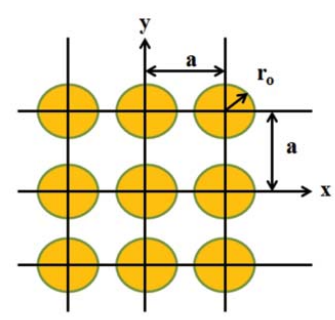

(b)

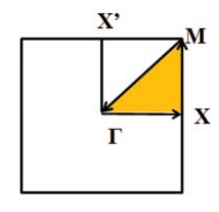

(c)

Figure 1. (a) Two dimensional square photonic crystal of circular rods of radius $r$ with lattice constant $a$; (b) transverse cross section of two-dimensional square lattice; (c) the shaded area is the irreducible Brillouin zones under study. 
study has the parameters with the column radius of $23 \mathrm{~mm}$ and the lattice size of $\mathrm{a}=\mathrm{b}=$ $50 \mathrm{~mm}$ for an array of circular SbSI cylinders embedded in an air background material. This results in a filling ratio of approximately $66 \%$. The phononic material parameters used in the calculations are $\rho_{S b S I}=5200 \mathrm{~kg} / \mathrm{m} 3, \rho_{\text {Air }}=1.3 \mathrm{~kg} / \mathrm{m} 3, c_{S b S I}=3200 \mathrm{~m} / \mathrm{s}$, and $c_{\text {Air }}=340 \mathrm{~m} / \mathrm{s}$. The unit cell of the system is a square lattice in Brillouin zone and its first irreducible Brillouin zone corresponding of this periodic structure is shown in Fig 1.

The $n_{x}$ and $n_{y}$ values in the reciprocal lattice vector $G$ are permitted to take the integer values from -9 to +9 (361 plane waves). Within an acceptable range, a reasonably good convergence was obtained. In order to show the feature apperaring in the band structures of the proposed structure, we illustrate the dispersion curves for a typical circular hole with a $\mathrm{r} / \mathrm{a}=0.46$ in Fig 2 which corresponds to $\% 66$ filling ratio. The plots are given in terms of the frequency, which is the vertical axis and the horizontal axis is the reduced wave vector. All the parameters are independent of $\mathrm{z}$ direction. That means $2 \mathrm{D}$ phononic crystal structure is invariant along the $\mathrm{z}$ direction. The first eighteen bands for propagation in the $(\mathrm{x}, \mathrm{y})$ plane were computed while the irreducible triangle $\Gamma-\mathrm{X}-\mathrm{M}-\Gamma$ of the Brillouin zone along its pheriphery was scanned, For band structure calculations, moving along the high symmetry axes of the first Brillouin zone was good enough. Figure 2 shows full band gaps along the $\Gamma-\mathrm{X}-\mathrm{M}-$ $\Gamma$ direction. Following the path along the $\Gamma-\mathrm{X}-\mathrm{M}-\Gamma$ direction, the first band gap appears between [0.4977-0.2666] kHz, the second one is between [1.6952-1.6253] kHz, the third one is between [1.9607-1.8499] kHz, and the fourth one occurs between [2.146-2.0759] $\mathrm{kHz}$, respectively. As it can be seen, the first band has the broadest energy gap in Fig 2.

The filling fraction in the structure is also changed by varying the hole radius to analyze the sensitivity of the bandwidth to the change of radius $r$. We must note that there is a minimum fill fraction of at least about $36 \%$ in order for a band to appear.

The density of states as a function of frequency or energy is also computed and the figure on the right panel in Figure 2 shows the corresponding phononic density of states (DOS) as a function of frequency. The phononic density of states were calculated by sampling k-points in the two dimensional BZ. We found the density of states as a function of frequency $\omega$ by doing a sum of all bands over all frequencies. DOS calculations are particularly important

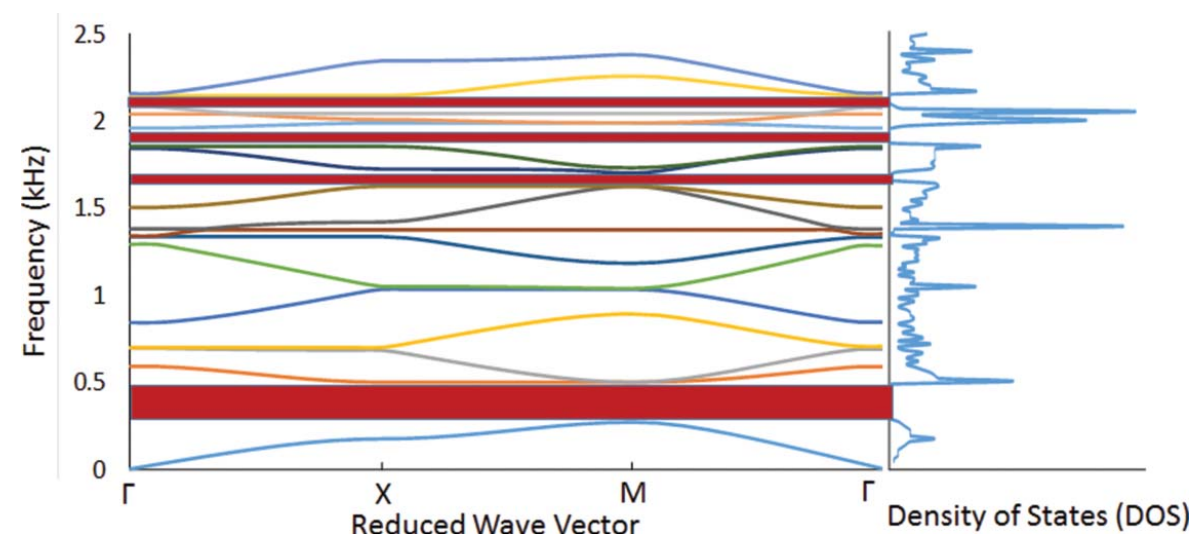

Figure 2. Acoustic band structures of infinite PnCs composed of SbSI rods in air. The lattice constant, radius of SbSI rods and filling fraction are $a=50 \mathrm{~mm}, r=23 \mathrm{~mm}, \mathrm{f}=66 \%$ respectively. The dashed region represents the complete stop band. The phononic density of states in PnC crystal. 
since some physical properties, such as thermal conductivity and specific heat, can be modified by changing the phonon density of states in a phononic crystal. Briefly, they provide insight into both microscopic and macroscopic properties.

\section{Equi-frequency surface}

Since the momentum conservation law is satisfied at the reflection and refraction of waves, it is convenient to analyze the reflection and refraction of a certain wave in the space of wave numbers by introducing the equi-frequency surface of the wave. This surface is directly described by the dispersion relation of the anisotropic medium at the fixed frequency $\omega$. Then, the group velocity of the wave in an anisotropic medium can be found as the frequency gradient in the space of wave vectors [28].

It is known that for a wave propagating in an isotropic medium with a fixed frequency $\omega$, the equi-frequency surface represents a sphere. In this case, the wave vector and group velocity vector, which determines the ray direction, are always parallel. However, equi-frequency

(a)

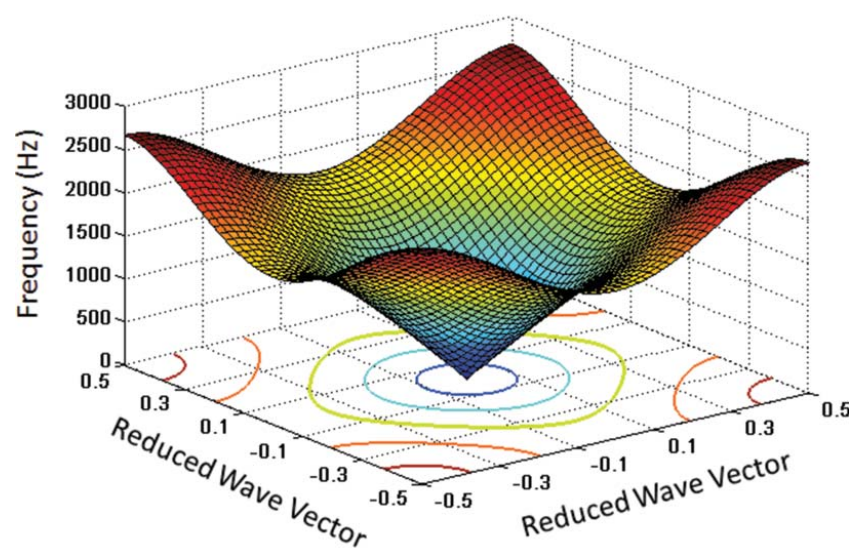

(b)

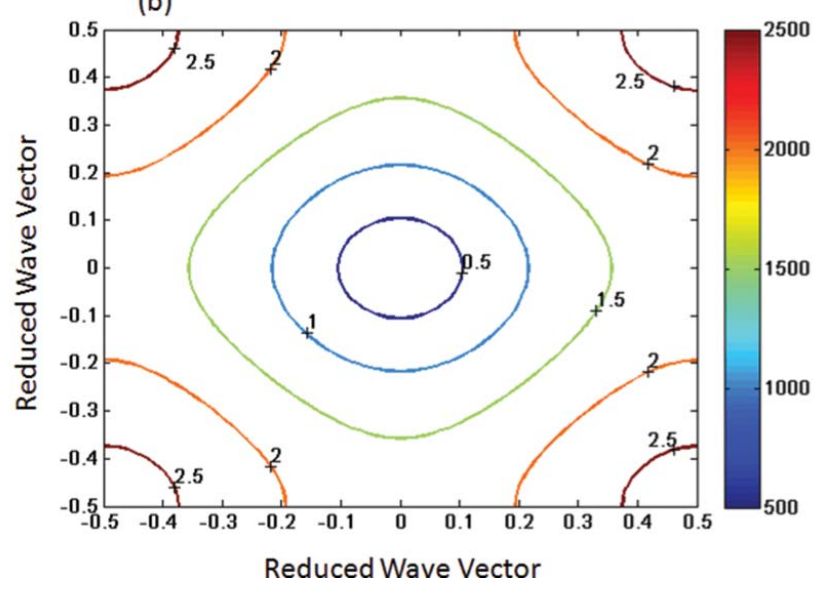

Figure 3. (a) Dispersion curve and (b) equifrequency contours for the first mode of the square PnC, calculated for all k-vectors in the first Brillouin zone, respectively. 
surface is not spherical for anisotropic media and the vectors are not parallel. By analogy with $3 \mathrm{D}$ case, the propagation, reflection, and refraction of the wave in $2 \mathrm{D}$ structure can be described in terms of the equi-frequency dependence which can be considered as the section of the dispersion surface in the space of variables by the plane corresponding to constant frequency. It is well known that the analysis of equi-frequency dependences is most efficient in the studies of $2 \mathrm{D}$ geometries, especially in solving problems when only orientations of the vector of incident, reflected, and refracted waves are of interest, and are not the amplitudes of the reflected and refracted rays. The equi-frequency dependence has a simple physical meaning

(a)

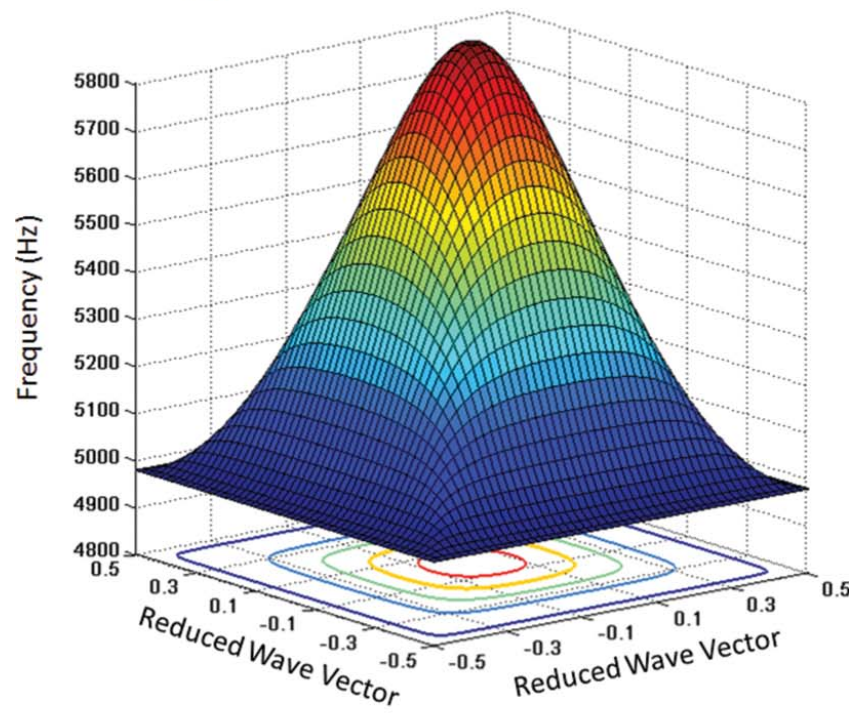

(b)

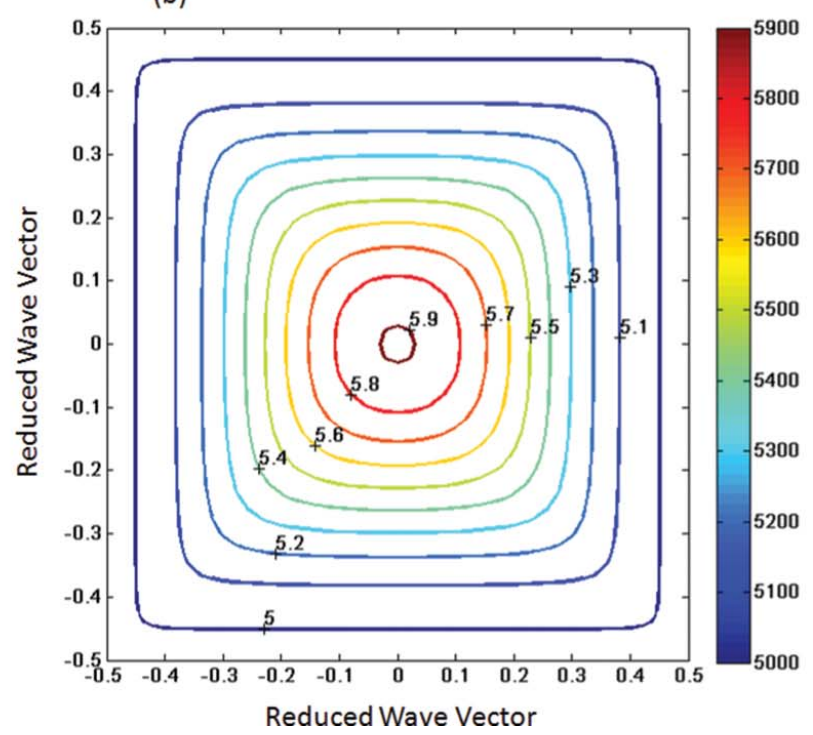

Figure 4. (a) Dispersion curve and (b) equifrequency contours for the second mode of the square PnC, calculated for all k-vectors in the first Brillouin zone, respectively. 
for the analysis of 2D geometries: since this dependence describe all the possible waves with the given frequency $\omega$ and various wave vectors, the directions of the reflected and the refracted rays can be determined by simply finding the points in equi-frequency dependences of media that satisfy the momentum conservation law at a known orientation of the boundary and a given angle of incidence of the wave. Now, we present some numerical examples for our PC structures. In all of these examples, we exploit symmetry to calculate the equi-frequency surfaces over the irreducible Brillouin zone of the entire Brillouin zone. First, we consider the equi-frequency surface of a square lattice of SbSI layers in air medium. Here, the map was discretized using 441 points per edge of the unit cell for the first band in Figure 3 (a). The map was discretized using 441 points per edge of the unit cell for the next band in Figure 4(a). The curves shown correspond to equi-frequency surfaces of the lowest order band up to frequencies just below the band gap starting at around $0.26 \mathrm{kHz}$. For the next band, the curves shown correspond to equi-frequency surfaces of the second lowest order band up to frequencies just below the band starting at around $0.50 \mathrm{kHz}$. Each point on the surface gives the possible eigen solutions that consist of all allowed wave vectors in the first Brillouin zone. Therefore, the plots retain detailed information but they are difficult to analyze. Figure 3 (b) and Figure 4(b) show contours of dispersion surfaces with values on the contours given. The equi-frequency contours are something that is more meaningful and easier to analyze. The plots basically indicate the contours of the energy flow which may allow us to identify irregularities in refraction, and they are also very helpful in understanding propagation effects within the crystal. In Figure 3(b) some contours are nearly circular and they imply that propagation of the energy is radially symmetric (no dependence on direction). The first and other bands of the acoustic waves are adopted to analyze the refractive angles and intensities of the wave transmission. Deviation from the circular shape depends on the radial amplitude which is a function of the incident frequencies. However, in Figure 4(b), first contour closest to center appears to be a circle, and then after a few contours deviations from circular shapes are observed for points away from the center. The circular shapes change into a square like contours, and a simple symmetry takes place for values of $\mathrm{kx}$ equal to ky through the square shaped ones.

\section{Conclusions}

The band gap and transmittance properties of a square lattice phononic crystal consisting of SbSI cylindrical rods in air were investigated using the plane wave expansin method by imposing elastic rigidity. In light of the calculations, band gap structures give the required physical conditions for possible uses as a possible acoustic/elastic filters, noise control or an insulator.

\section{Funding}

This work is supported by the projects DPT-HAMIT and NATO-SET-193, and one of the authors (Ekmel Ozbay) also acknowledges partial support from the Turkish Academy of Sciences.

\section{References}

1. Y. Pennec, B. Djafari-Rouhani, H. Larabi, J. Vasseur, A. C. Hladky-Hennion, Phononic crystals and manipulation of sound. Phys. Status Solidi. 6(9), 2080-2085 (2009). 
2. J.H Sun, T. T. Wu, Propagation of surface acoustic waves through sharply bent two-dimensional phononic crystal waweguidesusing a finite-difference time-domain method. Phys. Rev. B. 74(17), 174305 (2006).

3. J. Gao, J. C. Cheng, B. W. Li, Propagation of Lamb waves in one-dimensional quasiperiodic composite thin plates. A split of phonon band gap Appl. Phys. Lett. 90, 111908 (2007).

4. S. Mohammadi, A. A. Eftekhar, A. Khelif, W. D. Hunt, A. Adibi, Evidence of large high frequency complete phononic band gaps in silicon phononic crystal plates. Appl. Phys. Lett. 92(22), 221905 (2008).

5. X. F. Zhu, T. Xu, S. C. Liu, J. C. Cheng, Study of acoustic wave behavior in silicon-based onedimensional phononic-crystal plates using harmony response analysis. J. Appl. Phys. 106(10), 104901 (2009).

6. C. J. Rupp, M. L. Dunn, K. Maute, Switchable Phononic Wave Filtering, Guiding, Harvesting, and Actuating in Polarization-Patterned Piezoelectric Solids. Applied Physics Letters 96(11), 111902 (2010).

7. S. Jia-Hong, J. Jyun-Hua, Study of Surface Acoustic Waves in $\mathrm{SiO} 2 / \mathrm{LiNbO} 3$ Layered-Structure Phononic Crystals. Proceedings of Symposium on Ultrasonic Electronics (20-22 November): 34, 193-194 (2013).

8. A. H. Safavi-Naeini and O. Painter, Design of optomechanical cavities and waveguides on a simultaneous bandgap phononic-photonic crystal slab. Optics Express 18(14), 14926-14943 (2010).

9. T. Kurose, K. Tsuruta, C. Totsuji and H. Totsuji, Negative Refraction of Acoustic Waves in a TwoDimensional Phononic Crystal via FDTD Simulation. Materials Transactions 50(5), 1004-1007 (2009).

10. T. T. Wu, W. S. Wang, J. H. Sun, J. C. Hsu, Y. Y. Chen, Utilization of phononic-crystal reflective gratings in a layered surface acoustic device. Appl. Phys. Lett. 94(10), 101913 (2009).

11. I. F. Olsson III, R. H. El-Kady, M. F. Su, M. R. Tuck, J. G. Fleming, Microfabricated VHF acoustic crystals and waveguides. Sens. Actuators A. 145, 87-93 (2008).

12. T. T. Wu, Y. T. Chen, J. H. Sun, SCS Lin, T. J. Huang, Focusing of the lowest antisymmetric Lamb wave in a gradient-index phononic crystal plate. Appl. Phys. Lett. 98, 171911 (2011) 2011.

13. R. Lucklum, Phononic crystals and metamaterials - Promising new sensor platforms. Procedia Engineering. 87, 40-45 (2014).

14. P. Wang, L. Lu, K. Bertoldi, Topological Phononic Crystals with One-Way Elastic Edge Waves. Phys. Rev. Lett. 115, 104302 (2015).

15. Y. Tanaka, S. I. Tamura, Surface acoustic waves in two-dimensional periodic elastic structures. Physical Review B. 58, 7958-7965 (1998).

16. M. S. Kushwaha, P. Halevi, L. Dobrzynski, B. Djafari-Rouhani, Acoustic Band Structure of Periodic Elastic Composites. Phys. Rev. Lett. 71, 2022-2025 (1993).

17. W. M. Kuang, Z. L. Hou, Y. Y. Liu, H. Li, The bandgap of a photonic crystal with triangular dielectric rods in a honeycomb lattice. J. Opt. A-Pure Appl. Opt. 7, 525-528 (2005).

18. I. Olsson III, R. H. El-Kady, Microfabricated phononic crystal devices and applications. Measurement science and technology 20, 1-13 (2009).

19. E. I. Gerzanich, V. A. Lyakhovitskaya, V. M. Fridkin, B. A. Popovkin, SBSI and Other Ferroelectric AVBVICVII Materials. Current Topics in Materials Science. North-Holland Publishing Co. 1982; 10.

20. T. Inushima and K. Uchinokura, Anomalous Piezoelectric Properties of Ferroelectric Semiconductor SbSBr. Ferroelectrics 286(1), 119-129 (2003).

21. C. Xu, F. Cai, S. Xie, F. Li, R. Sun, X. Fu, R. Xiong, Y. Zhang, H. Zheng, J. Li, Phononic Crystal Tunable via Ferroelectric Phase Transition. Phys. Rev. Applied 4, 034009 (2015).

22. M. S. Kushwaha, P. Halevi, L. Dobrzynski, B. Djafari-Rouhani, Acoustic Band Structure of Periodic Elastic Composites. Phys. Rev. Lett. 71, 2022-2025 (1993).

23. A. Ruffa, Acoustic Wave Propagation Through Periodic Bubbly Liquids. J. Acoust. Soc. Am. 91(1), 1-11 (1992).

24. M. Sigalas, E. Economou, Elastic and Acoustic Wave Band Structure. J. Sound Vib. 158(2), 377382 (1992). 
25. M. Sigalas, E. Economou, Band Structure of Elastic Waves in Two Dimensional Systems. Solid State Commun. 86(3), 141-143 (1993).

26. R. Martinez-Sala, J. Sancho, J.V Sanchez, V. Gomez, Llinares, J., Meseguer, F., Sound Attenuation by Sculpture. Nature. 378, 241-242 (1995).

27. D. P. Elford, Band gap formation in acoustically resonant phononic crystals. PhD thesis. Loughborough University Institutional Repository 2010.

28. E. H. Lock, The properties of isofrequency dependences and the laws of geometrical optics. Phys. Usp. 51(4), 375-393 (2008). 